\title{
Right Ventricular Thrombus and Tricuspid Valve Dysfunction in a Patient with Behçet's Disease
}

\author{
Federica Valente1, Christian Motet $^{2}$, Stefan Rusu ${ }^{3}$, Frederic Vandergheynst ${ }^{4}$ \\ ${ }^{1}$ Department of Cardiology, Erasme University Hospital, Université Libre de Bruxelles, Brussels, Belgium \\ ${ }^{2}$ Department of Internal Medicine, CHU Tivoli, Université Libre de Bruxelles, Brussels, Belgium \\ ${ }^{3}$ Pathology Department, Erasme University Hospital, Université Libre de Bruxelles, Brussels, Belgium \\ ${ }^{4}$ Department of Internal Medicine, Erasme University Hospital, Université Libre de Bruxelles, Brussels, Belgium \\ Email: Federica.valente@ulb.be
}

How to cite this paper: Valente, F., Motet, C., Rusu, S. and Vandergheynst, F. (2020) Right Ventricular Thrombus and Tricuspid Valve Dysfunction in a Patient with Behçet's Disease. Case Reports in Clinical Medicine, 9, 319-328.

https://doi.org/10.4236/crcm.2020.910045

Received: September 1, 2020

Accepted: October 18, 2020

Published: October 21, 2020

Copyright (c) 2020 by author(s) and Scientific Research Publishing Inc. This work is licensed under the Creative Commons Attribution International License (CC BY 4.0).

http://creativecommons.org/licenses/by/4.0/

\begin{abstract}
Background: Behçet's disease (BD) is a multisystemic, chronic inflammatory disorder with a broad range of manifestations including within the cardiovascular system. Cardiac involvement like intracardiac thrombus (ICT) and valvular involvement in $\mathrm{BD}$ are rarely seen entities and often associated with poor prognosis. Case Presentation: We present the case of a young patient with vascular-Behçet diagnosed by the presence of intracardiac thrombus in the right ventricle, unresponsive to medical treatment. Even though the intracardiac lesion was successfully treated by surgical excision, he presented a severe tricuspid dysfunction some years later due to the extension of fibrosis with no surgical therapeutic option. Conclusion: Intracardiac thrombosis is a rare but early manifestation of Behçet's disease, which is difficult to diagnose and has a poor prognosis.
\end{abstract}

\section{Keywords}

Behçet's Disease, Cardiac Involvement, Intracardiac Thrombosis, Tricuspid Valve Regurgitation, Endomyocardial Fibrosis

\section{Introduction}

Behçet's disease is a chronic, relapsing, systemic disorder characterized by recurrent oral and genital ulcers, uveitis, acneiform lesions, vascular involvement and other clinical manifestations in multiple organ systems [1] [2] [3].

Vascular involvement is a common complication of BD affecting up to $40 \%$ [4] [5] [6] [7] of patients. Vascular manifestations particularly affect young men, 
during the first years following the onset of the disease. Venous complications are the most frequent vascular complications, affecting $14 \%$ to $40 \%$ of BD patients, followed by arterial complications ( $2 \%$ to $17 \%$ of $\mathrm{BD}$ patients).

Cardiac involvement has been reported infrequently in $\mathrm{BD}$ patients (1\% to $6 \%$ of patients) and may occur in the form of intracardiac thrombus, endocarditis, myocarditis, pericarditis, endomyocardial fibrosis, coronary arteritis, myocardial infarction, and valvular disease.

Although their frequency in Behçet's disease is very low, intracardiac thrombosis, endomyocardial fibrosis and valve dysfunction may be seen during the course of the disease and may lead to serious clinical consequences [8] [9] [10].

Here we report a case of a man with Behçet's disease with unusual manifestations and rare complications.

\section{Case Report}

A 25-year-old Caucasian man was admitted to our hospital in January 2014 with a history of progressive dyspnea for about 6 months, cough, and hemoptysis of unknown origin.

He had suffered from recurrent oral ulcers and acneiform lesions since adolescence, deep peripheral vein thrombosis in the lower extremities in 2011 and pulmonary embolism in July 2013, while being on anticoagulant treatment by acenocoumarol.

On physical examination, there were no signs of heart failure and no pathological pulmonary sounds; he presented aphthous oral lesions, but no signs of any other system involvement or fever.

Abnormal biochemical and hematological parameters included a hemoglobin concentration of $11 \mathrm{~g} / \mathrm{dl}$, an erythrocyte sedimentation rate of $59 \mathrm{~mm} /$ hour and a protein-C-reactive of $34 \mathrm{mg} / \mathrm{L}$. Liver and kidney function tests were normal. The work-up for a prothrombotic state including protein $\mathrm{C}$ deficiency, protein $\mathrm{S}$ deficiency, decreased antithrombin III activity, anticardiolipin and antiphospholipid antibodies and factor $\mathrm{V}$ Leiden was negative.

Echocardiographic examination showed an abnormal echogenic structure protruding in the cavity of the right ventricle $(38 \times 16 \mathrm{~mm}$ ) (Figure 1$)$. Transoesophageal echocardiography confirmed the presence of a mass adjacent to the lateral wall of the right ventricle under the tricuspid valve annulus, linked to another structure under the pulmonary valve, and a possible structure at the entrance site of the inferior vena cava to the right atrium (Figure 2). Heart valves were normal, as well as the left ventricle in size and their functions.

Cardiac MRI showed a right intraventricular mass with discrete early and late enhancement. No process infiltrating the myocardial wall was present (Figure 3).

Based on this data, the differential diagnosis included thrombus, tumoral lesion (malignant tumor or myxoma) and endocarditis.

Computed tomography showed chronic pulmonary embolismsequelae, 


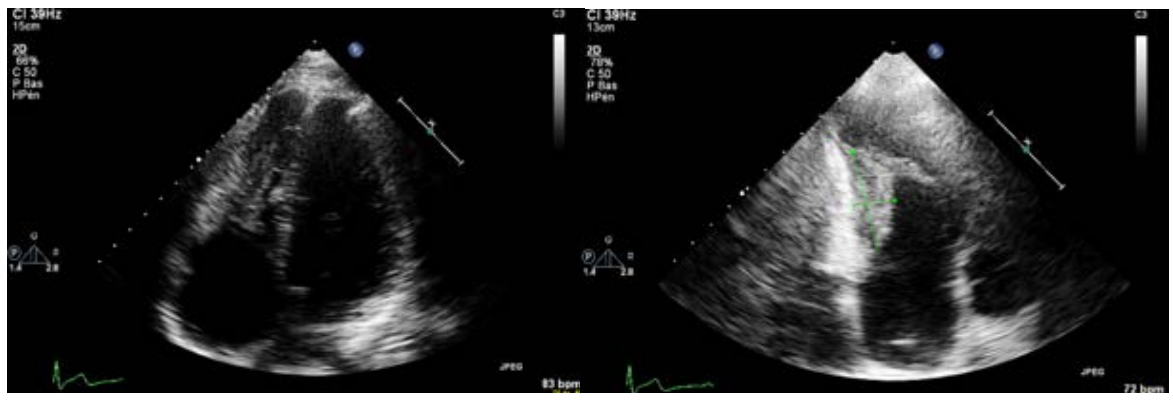

Figure 1. Transthoracic echocardiography on apical four chamber, showing an abnormal echogenic structure protruding in the cavity of the right ventricle $(38 \times 16 \mathrm{~mm})$.

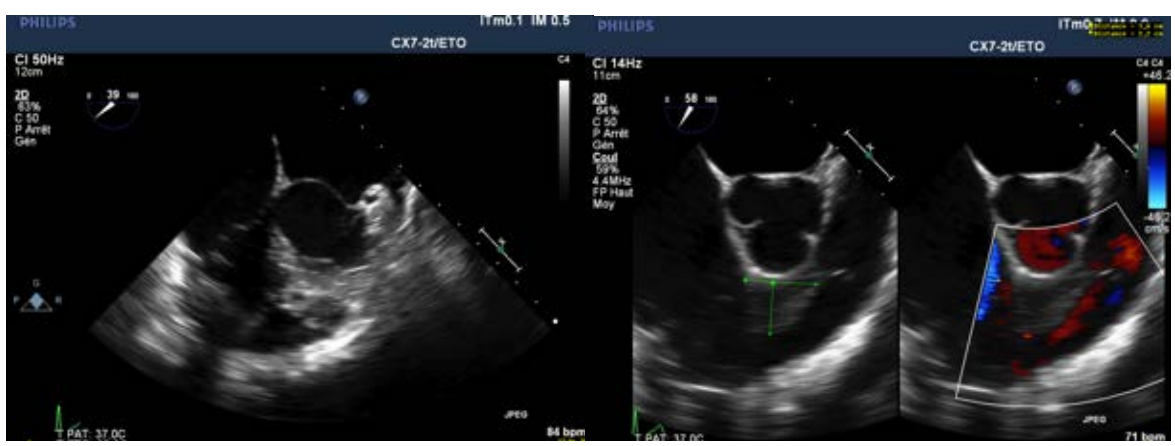

(a)

(b)

Figure 2. Transesophageal echocardiographic images. (a) Mass in the right ventricle. (b) Mass under the pulmonary valve.

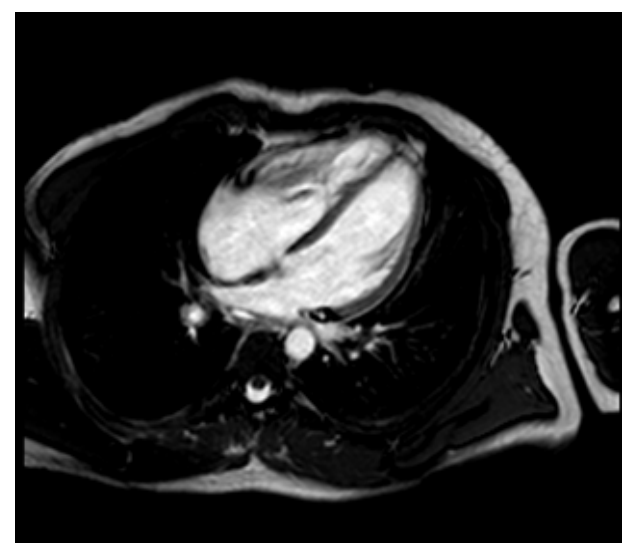

Figure 3. Cardiac magnetic resonance image showing right intraventricular cardiac mass.

pulmonary infarct in the right inferior lobe and partial thrombosis of the pulmonary veins. Pulmonary scintigraphy showed multiples perfusion defects (in the superior and inferior right lobe).

$\mathrm{PET} / \mathrm{CT}$ revealed a hypermetabolic abnormality in the right ventricle, but no other suspected lesions.

Repeated blood and urine cultures, immunoelectrophoresis, serological investigations, skin tests, bone marrow aspiration, and biopsy did not provide any evidence of bacterial endocarditis, malaria, fungal infections, tuberculosis, or 
malignancy. No arguments either for an autoimmune disease were present (antinuclear antibody, anti-DNA tests, ANCA were negative). HLA-B51 and pathergy tests were negative.

The diagnosis of Bechet's disease was based on the criteria of the International Criteria for Bechet's disease (ICBD) of 2013 [2] [3]. The patient had recurrent oral ulcerations, typical skin lesions (pseudofolliculits) and vascular complications.

While being investigated, the patient was treated with high dose intravenous heparin for 20 days, during which time the thrombi did not resolve. An anticoagulation treatment by antivitamin $\mathrm{K}$ was continued. After the diagnosis was made, treatment was initiated with high doses of methylprednisolone, azathioprine, and colchicine.

After one year of treatment, the thrombus dimensions only slightly decreased at echocardiographic controls. Therefore, the immunosuppressive treatment was changed, with a shift from azathioprine to cyclophosphamide (CYC) intravenous pulses. The patient achieved four pulses of CYC, however, the intracavitary mass persisted.

A surgical procedure was then performed in November 2015 to remove the mass and to enable a histological confirmation about the nature of the mass.

Behind the septal leaflet of the tricuspid valve, a fibrous thickening of the myocardium, was found and excised. No other suspected lesions were identified during the surgery.

The pathological investigation confirmed the presence of an organized thrombus, associated with endocardium thickening and fibrosis, extending to the underlying myocardium (Figure 4).

After the thrombectomy, with ongoing immunosuppressive treatment and anticoagulation, the patient showed neither inflammatory activity of $\mathrm{BD}$, nor

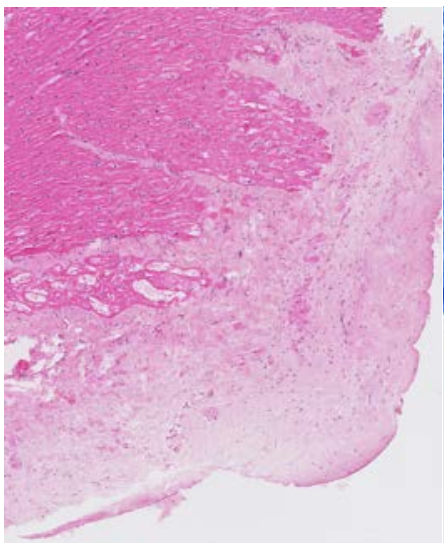

(a)

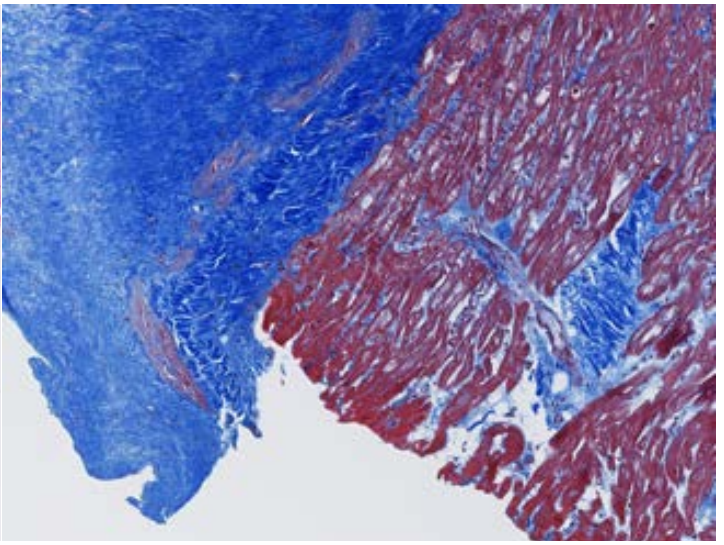

(b)

Figure 4. Pathology of the cardiac mass. (a). Significant thickening of the endocardium with fibrosis. Strands of fibrosis are extending to the underlying myocardium (Hematoxylin and Eosin staining-magnification $5 \times$ ). (b). Significant fibrotic thickening of the endocardium and patchy involvement of the myocardium: connective tissue-collagen in blue and cardiac muscle in red (Masson's trichrome staining-magnification 10x). 
recurrence of intracardiac thrombus for some years.

However, he had no improvement of his dyspnea. The echocardiography in 2019 showed a severe tricuspid regurgitation, due to annular dilation on the loss of coaptation of valvular leaflets; the posterior leaflet and the moderator band presented an important thickening, suggestive of fibrosis (Figure 5). The right atrium was severely dilated and subsequently the right ventricle was dysfunctional.

A right heart catheterization showed no pulmonary hypertension.

We concluded that the severe tricuspid regurgitation, due to a fibrosing process with restrictive physiology, was the cause of dyspnea.

No surgical option of valve repair or replacement could be proposed due to the high surgical risk (in particular of creating an obstruction of the right ventricle) and the limited benefits of a dysfunctional right ventricle with fibrosis.

After a multidisciplinary discussion, the chosen treatment was cardiac transplantation.

The patient accepted this solution and he has been listed for cardiac transplantation since May 2020.

\section{Discussion}

Originally described as a clinical syndrome of recurrent oral and genital ulcers and relapsing uveitis, Behçet's disease is now recognized as a chronic multisystem illness involving many organs [1] [2] [3].

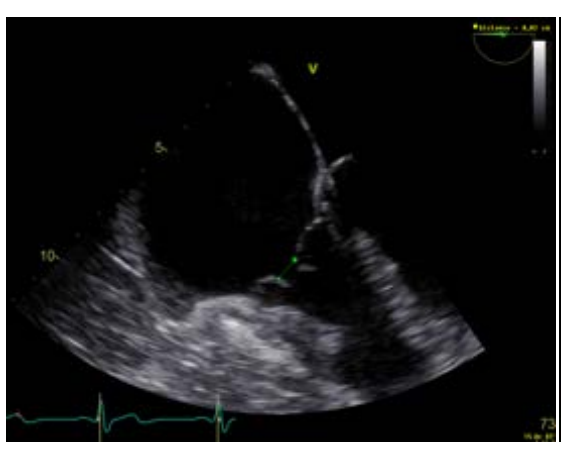

(a)

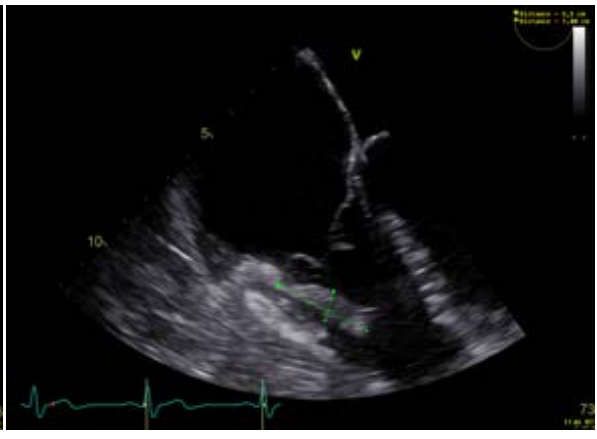

(b)

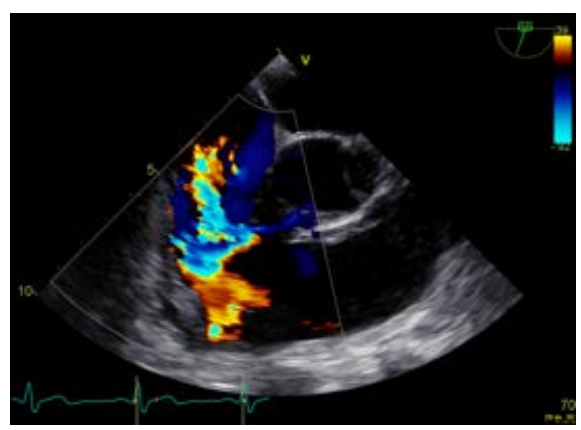

(c)

Figure 5. Transesophageal echocardiography. (a) Loss of coaptation of tricuspid valvular leaflets. (b) Thickening of the moderator band. (c) Severe tricuspid regurgitation. 
Vascular involvement is a common complication of BD affecting up to $40 \%$ [4] [5] [6] [7] of patients and is the most common cause of morbidity and mortality.

The concept of vasculo-Behçet has been adopted for cases in which vascular complications dominate the clinical features.

Venous involvement is the most frequent vascular complication, affecting 14 to $40 \%$ of BD patients [11], followed by arterial complications ( $2 \%$ to $17 \%$ of $\mathrm{BD}$ patients).

There is little data on cardiac involvement in BD; it has been reported in $1 \%$ to $6 \%$ of $\mathrm{BD}$ patients [8] [9] [10].

Cardiac abnormalities in BD include pericarditis, endocardial lesions (aortic regurgitation and less often mitral insufficiency), myocardial lesions (myocardial infarction, myocarditis and endomyocardial fibrosis) and intracardiac thrombosis [8] [9] [10].

Intracardiac thrombus (ICT) is an uncommon complication of BD [9] [10] [12] [13] [14] [15]. Since the first description, to date, less than 100 cases have been reported.

As described for other vascular manifestations, cardiac thrombosis affects particularly young men, coming from the Mediterranean basin regions or the Middle East, and may be the first manifestation of BD.

ICT mostly involves the right ventricle and is often associated with other venous thromboses (pulmonary embolism in $60 \%$ of cases and extension to the inferior vena cava in $40 \%$ of patients) [9] [10] [12] [13] [14] [15].

The clinical presentation of ICT is nonspecific in the majority of patients: fever, haemoptysis, dyspnoea, and a cough are usually the predominant symptoms [12]-[20].

Echocardiographic findings are not specific and could be indistinguishable from other lesions.

A comprehensive work-up is then needed to differentiate ICT from cardiac tumors such as myxoma and right-sided endocarditis.

In their retrospective study, Wang et al. [14] propose some typical echocardiographic features of ICT in $\mathrm{BD}$, such as: 1) involvement of the ventricle rather than the atria and mostly in the right heart; 2) often multiple; 3) usually hyperechoic and homogeneous with well-delineated borders; 4) usually immobile with a broad-based attachment to the atria or ventricle.

Additional imaging modalities that can also be used for diagnosis include cardiac MRI [14], which differentiate the thrombus, avascular without contrast uptake, from surrounding myocardium or tumors.

Since there are a limited number of ICT cases in BD, there is no consensus on the antiaggregant, anticoagulant, thrombolytic, immunosuppressive or surgical treatments.

In the larger series of patients presenting intracardiac thrombosis, corticosteroids and/or other immunosuppressors were associated with complete resolution 
of the thrombus in most cases, suggesting that vascular inflammation plays a major role in thrombus formation [9] [10] [12] [13] [14] [15] [21].

Even though the use of anticoagulation is still controversial [22] due to potential haemorrhagic risk in patients with associated aneurysm [23], the majority of patients with ICT are anticoagulated.

Surgical removal is not the preferred choice because of the high rate of surgical complications and the risk of thrombosis recurrence. However, it should be considered in cases with unclear diagnosis, unresponsive to medical treatment or impaired hemodynamic caused by ICT [24] [25] [26] [27].

In our patient, an immunosuppressive treatment with azathioprine and methylprednisone, followed by cyclophosphamide was used for one year, as well as an anticoagulation therapy with acenocoumarol. In the absence of resolution of intracardiac thrombosis, surgery was proposed in order to confirm the diagnosis of thrombosis and to treat it.

The histopathological examination in our case showed organized thrombus and significant endocardial thickening with fibrosis, extending to the underlying myocardium.

The underlying mechanism of intracardiac mass formation in $\mathrm{BD}$ has not been fully clarified.

Cardiac thrombi in $\mathrm{BD}$ is located mainly in the right ventricle. The reason for predilection to involve RV is unclear. Based on autopsy findings, endomyocardial fibrosis likely plays a role in the intra-cardiac thrombus formation [28].

Endomyocardial fibrosis (EMF) may represent the sequelae of intracardiac thrombosis or vasculitis involving the endocardium or the myocardium.

In EMF, there is fibrosis of endocardial surfaces that leads to restrictive physiology. Additionally, tethering of the atrioventricular (AV) valve papillary muscles and/or fibrosis of the respective ventricular inflow tracts lead to significant AV valve regurgitation [29] [30] [31].

Four years after the thrombectomy, our patient developed a severe tricuspid regurgitation associated with right atrial dilatation and right ventricular dysfunction.

The valvular insufficiency was the consequence of the extension of fibrosis to the leaflets.

The management of these situations is controversial [30] [31].

In the rare cases reported amongst the literature of endomyocardial fibrosis complicated by cardiac failure, surgical excision with or without valvular intervention is suggested with favourable short-term results, but the long-term results are controversial.

In the absence of a surgical option for our patient, we proposed a cardiac transplantation.

In Behçet's disease, cardiac transplantation remains a challenge due to the risk of post-operatively complications on vascular and atrial anastomoses, caused by an excessive inflammatory response at puncture sites.

Two cases of successful heart transplantation in $\mathrm{BD}$ have been reported: one 
in a patient with end-stage heart failure due to multiples coronary aneurysms recurring after several interventions [32], the other for a Stanford type A aortic dissection [33]. It is unclear whether the immunosuppressive treatment to prevent rejection immunosuppression could impact the recurrence of vascular disease due to Behçet's disease.

\section{Conclusions}

In conclusion, intracardiac thrombosis is a rare but early manifestation of Behçet's disease.

The diagnosis could be tricky and surgical resection should be considered in cases with unclear diagnoses, which are not responding to medical treatment.

ICT is a cardiac complication with poor prognosis, due to the association or progression to endomyocardial fibrosis, leading to severe valvular and ventricle dysfunction.

\section{Conflicts of Interest}

The authors declare no conflicts of interest regarding the publication of this paper.

\section{References}

[1] Sakane, T., Takeno, M., Suzuki, N. and Inaba, G. (1999) Behçet's Disease. The New England Journal of Medicine, 341, 1284-1291. https://doi.org/10.1056/NEJM199910213411707

[2] (1990) Criteria for Diagnosis of Behçet's Disease. International Study Group for Behcet's Disease. The Lancet, 335, 1078-1080. https://doi.org/10.1016/0140-6736(90)92643-V

[3] Davatchi, F., Assaad-Khalil, S., Calamia, K.T., et al. (2013) The International Criteria for Behcet's Disease (ICBD): A Collaborative Study of 27 Countries on the Sensitivity and Specificity of the New Criteria. The Journal of the European Academy of Dermatology and Venereology, 28, 338-347.

[4] Düzgün, N., Ateş, A., Aydintuğ, O.T., Demir, Ö. and Ölmez, Ü. (2006) Characteristics of Vascular Involvement in Behçet's Disease. Scandinavian Journal of Rheumatology, 35, 65-68. https://doi.org/10.1080/03009740500255761

[5] Houman, M.H., Neffati, H., Braham, A., Harzallah, O., Khanfir, M., Miled, M., et al. (2007) Behçet's Disease in Tunisia. Demographic, Clinical and Genetic Aspects in 260 Patients. Clinical and Experimental Rheumatology, 25, S58-S64.

[6] Ideguchi, H., Suda, A., Takeno, M., Ueda, A., Ohno, S. and Ishigatsubo, Y. (2011) Characteristics of vascular Involvement in Behçet's Disease in Japan: A Retrospective Cohort Study. Clinical and Experimental Rheumatology, 29, S47-S53.

[7] Koç, Y., Güllü, I., Akpek, G., Akpolat, T., Akpolat, T., Kansu, E., Kiraz, S., et al. (1992) Vascular Involvement in Behçet's Disease. The Journal of Rheumatology, 19, 402-410.

[8] Wechsler, B., Du, L.T. and Kieffer, E. (1999) Cardiovascular Manifestations of Behcet's Disease. Annales de Médecine Interne (Paris), 150, 542-554.

[9] Geri, G., Wechsler, B., Thi Huong Du, L., Isnard, R., Piette, J.C., Amoura, Z., Resche-Rigon, M., Cacoub, P. and Saadoun, D. (2012) Spectrum of Cardiac Lesions 
in Behçet Disease: A Series of 52 Patients and Review of the Literature. Medicine (Baltimore), 91, 25-34. https://doi.org/10.1097/MD.0b013e3182428f49

[10] Desbois, A.C., Wechsler, B., Cluzel, P., Helftd, G., Boutina, D., Piette, J.-C., Cacoub, P. and Saadoun, D. (2014) Cardiovascular Involvement in Behçet's Disease. La Revue de Médecine Interne, 35, 103-111. https://doi.org/10.1016/j.revmed.2013.12.002

[11] Saadoun, D., Asli, B., Wechsler, B., Houman, H., Geri, G., Desseaux, K., et al. (2012) Long-Termoutcome of Arterial Lesions in Behcet Disease: A Series of 101 Patients. Medicine (Baltimore), 91, 18-24. https://doi.org/10.1097/MD.0b013e3182428126

[12] Mogulkoç, N., Burgess, I.M. and Bishop, P.W. (2000) Intracardiac Thrombus in Behçet's Disease. A Systematic Review. Chest, 118, 479-587.

https://doi.org/10.1378/chest.118.2.479

[13] Zhu, Y.L., Wu, Q.J., Guo, L.L. and Fang, L.G. (2012) The Clinical Characteristics and Outcome of Intracardiac Thrombus and Aortic Valvular Involvement in Behçet's Disease: An Analysis of 20 Cases. Clinical and Experimental Rheumatology, 30, S40-S45.

[14] Wang, H., Guo, X.X., et al. (2016) Intracardiac Thrombus in Patients with Behcet's Disease: Clinical Correlates, Imaging Features, and Outcome: A Retrospective, Single-Center Experience. Clinical Rheumatology, 35, 2501-2507.

[15] Emmungil, H., Yaşar Bilge, N.Ş., Küçükşahin, O., Kiliç, L., Okutucu, S., Gücenmez, S., Kalyoncu, U., Kaşifoğlu, T., Turgay, M. and Aksu, K. (2014) A Rare but Serious Manifestation of Behçet's Disease: Intracardiac Thrombus in 22 Patients. Clinical and Experimental Rheumatology, 32, S87-S92.

[16] Basaran, Y., Degertekin, M., Direskeneli, H. and Yakut, C. (2000) Cardiac Thrombosis in a Patient with Behcet's Disease: Two Years Follow-Up. The International Journal of Cardiovascular Imaging, 16, 377-382. https://doi.org/10.1023/A:1026584409912

[17] Gürgün, C., Sagcan, A., Cinar, C.S., Yagdi, T., Zoghi, M., Tekten, T., et al. (2000) Right Atrial Andventricular Thrombi in Behçet's Disease: A Case Report and Review of Literature. Blood Coagulation \& Fibrinolysis, 11, 107-110. https://doi.org/10.1097/00001721-200011010-00012

[18] Duchêne, F., Berthier, S., De Wazières, B., Zyka, F., Leroy, J., Estavoyer, J.M., et al. (1998) Behçet's Disease with Cardiac and Pulmonary Manifestations. La Presse Médicale, 1983, 1674-1676.

[19] Baykan, M., Çelik, S.Q. and Çakirbay, H. (2001) Behçet's Disease with a Large Intracardiac Thrombus: A Case Report. Heart, 85, e7.

https://doi.org/10.1136/heart.85.4.e7

[20] El-Ramahi, K.M., Fawzy, M.E., Sieck, J.O. and Vanhaleweyk, G. (1991) Cardiac and Pulmonary Involvement in Behcet's Disease. Scandinavian Journal of Rheumatology, 20, 373-376. https://doi.org/10.3109/03009749109096815

[21] Desbois, A.C., Wechsler, B., Resche-Rigon, M., et al. (2012) Immunosuppressants Reduce Venous Thrombosis Relapse in Behcet's Disease. Arthritis \& Rheumatism, 64, 2753-2760. https://doi.org/10.1002/art.34450

[22] Wechsler, B., Lê Thi Huong, D.-B. and Saadoun, D. (2009) EULAR Recommendations for the Management of Behcet's Disease: Evidence-Based or Experience-Based Medicine. La Revue de Médecine Interne, 30, 939-941.

https://doi.org/10.1016/j.revmed.2009.09.002

[23] Calamia, K.T., Schirmer, M. and Melikoglu, M. (2011) Major Vessel Involvement in Behçet's Disease: An Update. Current Opinion in Rheumatology, 23, 24-31. 
https://doi.org/10.1097/BOR.0b013e3283410088

[24] Darie, C., Knezinsky, M., Demolombe-Rague, S., Pinede, L., Perinetti, M., Ninet, J.F. and Ninet, J. (2005) Cardiac Pseudotumor Revealing Behcet's Disease. La Revue de Médecine Interne, 26, 420-424. https://doi.org/10.1016/j.revmed.2004.12.015

[25] Kirali, K., Civelek, A., Daglar, B., Sismanoglu, M., Akinci, E., Berki, T., Isik, O. and Yakut, C. (1998) An Uncommon Complication of Behcet's Disease: Intracardiac Thrombosis Needing Surgical Treatment. The Thoracic and Cardiovascular Surgeon, 46, 102-110. https://doi.org/10.1055/s-2007-1010201

[26] Mendes, L.A., Magraw, L.L., Aldea, G.S. and Davidoff, R. (1994) Right Ventricular Thrombus: An Unusual Manifestation of Behçet's Disease. Journal of the American Society of Echocardiography, 7, 438-440.

https://doi.org/10.1016/S0894-7317(14)80208-3

[27] Farouk, H. (2014) Behcet's Disease, Echocardiographers, and Cardiac Surgeons: Together Is Better. Echocardiography, 31, 783-787. https://doi.org/10.1111/echo.12524

[28] Lakpanhal, S., Tani, K., Lie, J.T., et al. (1985) Pathologic Features of Behçet's Syndrome: A Review of Japanese Autopsy Registry Data. Human Pathology, 16, 790-795. https://doi.org/10.1016/S0046-8177(85)80250-1

[29] Arslan, C., Arapi, B., Seyahi, E., Tel, C. and Tüzün, K.H. (2014) Right Ventricular Thrombus and Tricuspid Valve Dysfunction in A Patient with Behçet's Syndrome. Clinical and Experimental Rheumatology, 32, S109-S111.

[30] Huong, D.L., Wechsler, B., Papo, T., De Zuttere, D., Bletry, O., Hernigou, A., Delcourt, A., Godeau, P. and Piette, J.C. (1997) Endomyocardial Fibrosis in Behçet's Disease. Annals of the Rheumatic Diseases, 56, 205-208. https://doi.org/10.1136/ard.56.3.205

[31] Buturak, A., Saygili, O., Ulus, S., Kalfa, M., Karabulut, H., Alhan, C., Dagdelen, S. and Aksu, K. (2014) Right Ventricular Endomyocardial Fibrosis Mimicking Ebstein Anomaly in a Patient with Behçet's Disease: Case Report and Review of the Literature. Japan College of Rheumatology. Modern Rheumatology, 24, 532-536. https://doi.org/10.3109/14397595.2013.874731

[32] Hollander, S.A., Yasnovsky, J.R., Reinhartz, O., Chan, F., Sandborg, C., Hunt, S., Bernstein, D. and Chin, C. (2010) Behcet's Disease and Heart Transplantation: A Word of Caution. The Journal of Heart and Lung Transplantation, 29, 1306-1308. https://doi.org/10.1016/j.healun.2010.07.010

[33] Ren, W., Li, B.W., Wang, Z.W., Wu, Z.Y., Ruan, Y.L. and Wang, J.H. (2020) When Behcet's Disease Meets Stanford Type A Aortic Dissection, Heart Transplantation Is a Reliable Treatment. Annals of Translational Medicine, 8, 251.

https://doi.org/10.21037/atm.2020.01.43 